\title{
Impact of a quality improvement intervention on the incidence of unplanned extubations in a Pediatric Intensive Care Unit
}

\author{
Claudia N. Meregalli, M.D., ${ }^{a}$ Facundo A. Jorro Barón, M.D., Marcela A. \\ $D^{\prime}$ Alessandro, B.S. in Kinesiology, ${ }^{a}$, Esteban P. Danzi, B.S. in Kinesiology, ${ }^{a}$ and \\ Gustavo E. Debaisi, M.D. ${ }^{a}$
}

\begin{abstract}
Introduction. An unplanned extubation (UEX) is a frequent and preventable adverse event of mechanical ventilation.

Objectives. To find out the causes of unplanned extubation and assess the use of a quality improvement intervention to reduce this event rate.

Population. Patients with invasive mechanical ventilation for more than 12 hours over the period from May 1st 2010 and April 30th 2011 in a Pediatric Intensive Care Unit (PICU).

Design. Uncontrolled, before and after study. A quality improvement intervention was used over the period between November 2010 and April 2011, which included training PICU staff, establishing standards to fix the endotracheal tube and control its fixation.

Results. There were 395 patients admitted to the PICU, $262(66 \%)$ were on mechanical ventilation for more than 12 hours. A total of 27 patients had 30 UEX events: 22 during the period between May and October 2010, and 8 during the period between November 2010 and April 2011. The most common causes of UEX were incorrect fixation of the endotracheal tube $(n=11)$ and the use of a procedure of care $(n=11)$.

Patients with UEX required additional days on mechanical ventilation: 7 (3-15.5) versus 5 (2-8) days; $p=0.077$ and hospitalization: 9.5 (519) versus 7 (4-10) days; $p=0.049$. During the implementation of the quality improvement intervention, the mean incidence of UEX decreased from 2.30 (95\% CI: 1.12-3.49) to 0.86 (95\% CI: $0.27-1.44)$ per 100 days of mechanical ventilation $(\mathrm{p}=0.037)$.

Conclusions. The most common causes of UEX were the incorrect fixation of the endotracheal tube and the use of a procedure of care. The implementation of a quality improvement intervention reduced the incidence of UEX.

Key words: unplanned extubation, children, tracheal extubation, quality improvement, mechanical ventilation.
\end{abstract}

http:/ /dx.doi.org/10.5546/aap.2013.391

\section{INTRODUCTION}

Conflict of Interest: None.

Received: 12-20-2012 Accepted: 03-15-2013 life support techniques for treating critically ill children, endotracheal intubation and mechanical ventilation
(MV) have become a routine practice in the PICU. In most cases, the endotracheal tube (ETT) is electively removed once the cause leading to MV is resolved. A common and usually preventable adverse event of MV is the unplanned extubation (UEX), defined as the removal of the ETT at a time different from that selected for elective extubation. ${ }^{1}$ UEX increases morbidity and mortality in PICU patients and exposes them to emergency endotracheal intubation complications resulting from reintubation, thus prolonging the duration of MV and the length of stay in the PICU. ${ }^{2-6}$

Continuous quality improvement programs to reduce the incidence of UEX have demonstrated that training the PICU staff, identifying risk patients, standardizing procedures of care and developing sedation and analgesia and MV weaning protocols can actually decrease the incidence of UEX. ${ }^{1,2,7-10}$

The objectives of this study were to discover the causes of UEX in order to design a quality improvement intervention aimed at reducing UEX incidence and assess such intervention's effectiveness.

\section{MATERIAL AND METHODS Design}

Uncontrolled, before and after study. Approved by the Institutional Review Board of the Teaching and Research Department.

\section{Description of the study context}

Research was conducted at the 
"Pedro de Elizalde". This unit has 11 medical surgical beds, with no cardiovascular surgery. Orotracheal intubation was the most commonly used approach. The fixation technique was not standardized until the intervention was implemented; the tube was usually fixed with silk-like or common medical tape, one or two pieces across the upper lip. All patients with invasive MV were sedated with fentanyl and midazolam with continuous infusion; if required, neuromuscular-blocking drugs were used (vecuronium or pancuronium). During the study, the PICU did not have sedation, analgesia nor MV weaning protocols in place.

\section{Sample}

All children requiring endotracheal intubation for invasive MV for more than 12 hours in the period between May $1^{\text {st }} 2010$ and April 30 2011.

A minimum number of 800 days with MV per group was required in order to detect a difference $(p<0.05)$ in the UEX rate with a statistical power of more than $80 \%$, considering a reference rate of $<1$ UEX per 100 days of MV and an incidence of $>2$ UEX per 100 days of MV before the intervention.

\section{Data collection instrument}

Data were collected by direct observation of events. In order to avoid missing events and data at the moment of the UEX, progress notes and nurse records were reviewed on a daily basis.

The data collection record had three parts (see Annex 1 in electronic format). Unplanned extubation was defined as the removal of the ETT at a time different from that selected for elective extubation.1 UEX incidence was expressed as the number of UEX per 100 days of MV. The cause of the UEX and the corresponding definition were considered:

1) Use of procedures of care: these included washing the patient, changing the ETT fixation, aspirating secretions, taking an X-ray, changing the patient's position, and placing vascular accesses.

2) Incorrect ETT fixation: loose or detached adhesive tape favoring the migration of the ETT. ETT fixation is a procedure of care of the critically ill patient; it was considered separately from the other procedures of care due to its relevance as a cause of UEX.

3) Inadequate level of sedation: it was evaluated with the Ramsay score. An inadequate level of sedation is a patient with a level 1, and adequate is between 2 and $6 .^{11}$
4) Inadequate nurse-patient ratio: any ratio over $1: 2$.

5) Abundant secretions: more than three aspirations by 6-hour nurse shift.

6) MV weaning: transition between ventilation support and complete spontaneous breathing. ${ }^{12}$

7) Transfer: when UEX occurs while transferring the patient to the operating room or to the cath lab, or to have a diagnostic imaging test.

The research was carried out in two stages. During the first stage ("before"), from May to October 2010, baseline data were analyzed. During the second stage ("after"), from November 2010 to April 2011, a quality improvement intervention was implemented to reduce the incidence of UEX in the PICU.

Package of measures applied (see Annex 2 in electronic format):

- Training the PICU staff with the purpose of:

- Reporting the results of the first stage of the research.

- Creating awareness regarding the relevance and consequences of UEX.

- Disseminating the content of the package of improvement measures and how to implement it.

- Standardizing the ETT fixation process. The fixation technique and the material used (Tensoplast Sport ${ }^{\circledR}$ ) were standardized; in addition, data on the ETT size and depth were recorded on a card placed at the head of the patient's bed.

- Standardizing the ETT fixation monitoring. The correct fixation of the ETT was verified once per nurse shift and before washing the patient.

\section{Statistical analysis}

Data were analyzed using the STATA 10.1 (Statistics/Data Analysis Stata Corporation 4905 Lakeway Drive College Station, TX, USA) software. Values were expressed as mean and standard deviation or as median and interquartile range, Groups were compared using the Student's $t$ test or the Wilcoxon test by normal range. The $X^{2}$ test was used for dichotomous outcome measures. A value of $p<0.05$ was considered significant.

For the graphic comparison of UEX incidence between both periods, a u-control chart was used, taking \pm 2 standard deviations as the upper and lower control limits. 


\section{RESULTS}

During the first stage of the research, 232 patients were admitted to the PICU of the HGNPE; of them, 162 (70\%) were on MV for more than 12 hours. The total number of days of MV were 1026. Twenty two UEX episodes were recorded in 20 patients: 18 patients had 1 UEX, and 2 had 2 UEX. The mean incidence of UEX was 2.30 per 100 days of MV.

The most common causes were incorrect ETT fixation $(n=11)$ and the performance of a procedure of care $(\mathrm{n}=11)$ (Figure 1).

A single cause was identified only in 9 UEX; the remaining 13 UEX had multiple causes.

Procedures of care related to UEX included: patient washing $(n=5)$, performing an X-ray $(n=1)$, placing a vascular access $(n=1)$, changing the ETT fixation $(n=1)$, and procedure not recorded $(n=3)$.

The highest percentage of UEX (41\%) occurred during the morning nurse shift on working days (Figure 2).

FIGURE 1. Causes of unplanned extubation (UEX)

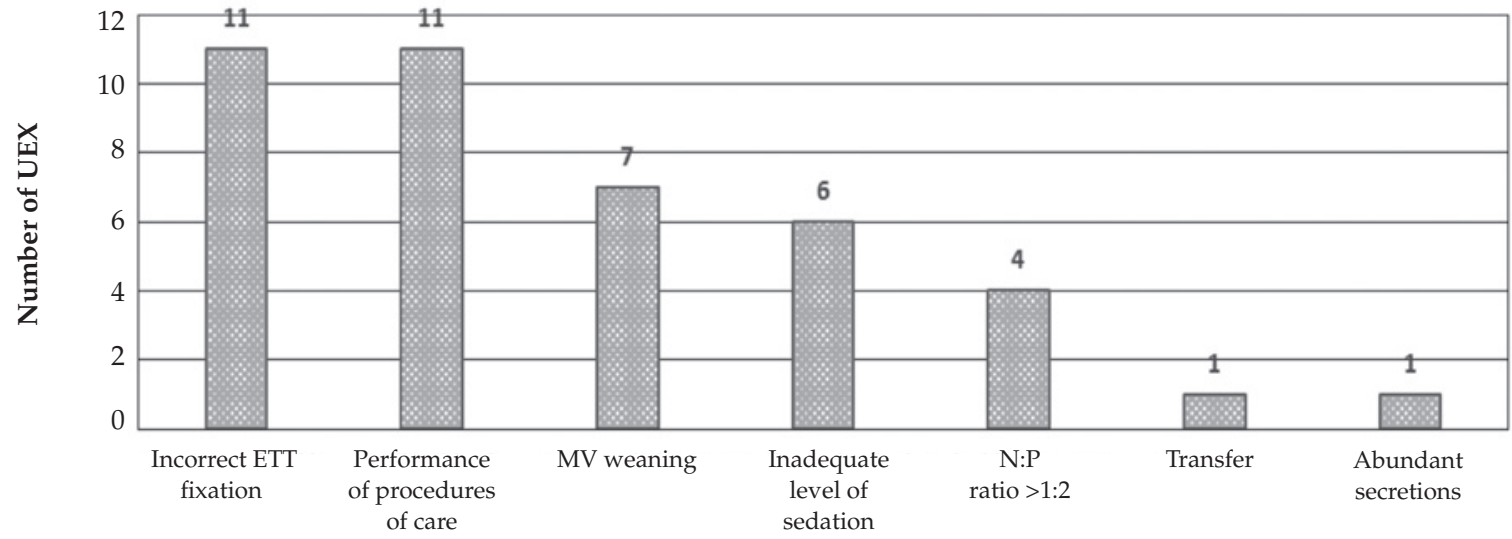

Causes of UEX

ETT: endotracheal tube

MV: mechanical ventilation

$\mathrm{N}: \mathrm{P}$ : nurse:patient

FIGURE 2. Distribution of unplanned extubations by nurse shift $(n=22)$

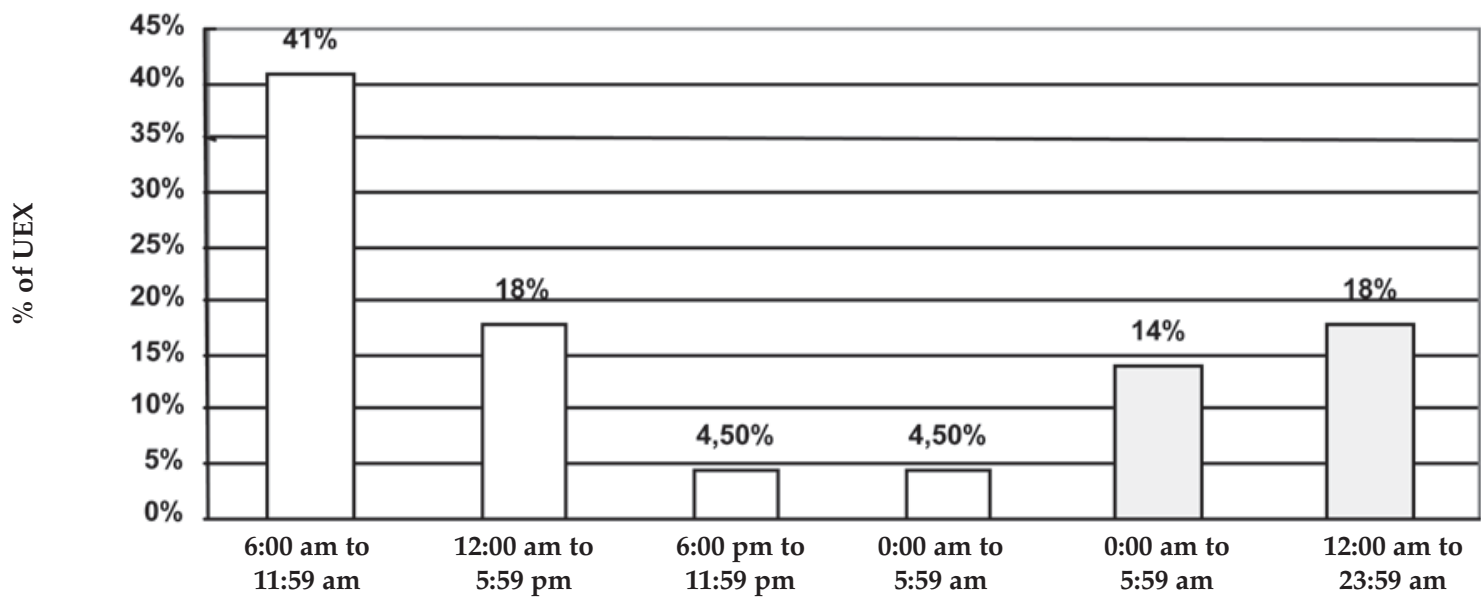

Nurse shifts 
Patients with UEX required more days of MV: 7 (IQ: 3-15.5) versus 5 (IQ: 2-8) days, $\mathrm{p}=0.077$; and a longer stay in the PICU: 9.5 (IQ: 5-19) versus 7 (IQ: 4-10) days, $p=0.049$. No deaths associated with UEX were recorded.

Out of the 22 UEX, $16(73 \%)$ required reintubation. All UEX occurring during complete ventilation support required reintubation, while only 1 UEX occurring during MV weaning required reintubation.

In total, $68 \%(n=15)$ of UEX occurred during complete ventilation support, while $32 \%(n=7)$ occurred during MV weaning; $68 \%$ between day 1 and 5 of starting MV. Patients with UEX and those with no UEX were of similar age, weight, and Pediatric Index of Mortality 2 (PIM2) (Table 1).

While the quality improvement intervention was applied, 163 patients were admitted to the PICU; of them, 100 (61\%) required MV for more than 12 hours. The total number of days of MV in this period was 847. Eight UEX episodes were recorded in 7 patients. The causes of UEX were as follows: MV weaning $(n=5)$, inadequate nurse-patient ratio $(n=4)$, inadequate level of sedation $(n=4)$, incorrect ETT fixation $(n=3)$, and performing a procedure of care $(n=1)$.

The mean UEX incidence was 0.86 per 100 days of MV, which was lower than the mean incidence rate before implementing the quality improvement intervention (2.30; 95\% CI: 1.12-3.49 versus 0.86; 95\% CI: 0.27-1.44, $\mathrm{p}=0.037$ ) (Figure 3). Patients before and after the implementation of the improvement strategy were similar, except for the PIM2, which was higher among patients admitted during the second research stage (3.6; IQ: 1.3-8.4 versus 7; IQ: 2.7-16.2, $\mathrm{p}=0.0007$ ) (Table 2).

The mortality rate among patients requiring MV for more than 12 hours during the study period was $9.5 \%(n=25)$.

\section{DISCUSSION}

UEX are common, and many times preventable, adverse events of MV. The incidence of UEX in the pediatric population in published studies over the past 20 years has varied between 0.11 and 2.7 UEX per 100 days of MV ${ }^{3,5}$

The incidence observed in the HGNPE's PICU during the first 6 months of study was 2.30 per 100 days of MV, similar to that reported by other studies in the stage prior to the implementation of the intervention to reduce UEX occurrence. ${ }^{2,4,7,13}$

There is no consensus regarding an acceptable incidence of UEX. A rate of zero may be desirable, but at the same time, dangerous. This objective could be achieved using high doses of sedation or extubating patients before they comply with the criteria for MV weaning, situations related to a poor clinical progress and requiring more days of MV and a longer stay in the PICU.

The causes associated with UEX include patient age, agitation and level of sedation, ETT fixation, amount of secretions, nurse-patient ratio, endotracheal intubation path, cause for admission to the PICU, physical restraint, procedure of care, transfer, and MV weaning. ${ }^{1-3,14}$

This analysis did not include among the causes of UEX the endotracheal intubation approach because all patients had received orotracheal intubation during the study period; the use of restraints because there is no scientific evidence supporting their use; and the cause of admission to the PICU because most authors agree that the lower incidence of UEX in patients admitted to the surgical PICU, the lower the number of days with MV. The most common causes associated with UEX in this unit were inadequate ETT fixation and the performance of procedures of care, which included washing the patient as the most predominant procedure.

TABLE 1. Demographic and clinical characteristics of the study population

\begin{tabular}{lccc}
\hline & $\begin{array}{c}\text { Patients with no UEX } \\
(\mathbf{n}=\mathbf{1 4 2})\end{array}$ & $\begin{array}{c}\text { Patients with UEX } \\
(\mathbf{n = 2 0})\end{array}$ & $\mathbf{p}$ \\
\hline Age (months), median (IQ) & $7(3-26)$ & $12(2-50)$ & 0.888 \\
Weight (kg), median (IQ) & $7.35(5-12)$ & $8.8(5.3-12)$ & 0.643 \\
PIM2, median (IQ) & $3.3(1.22-8.4)$ & $5.84(1.3-8.5)$ & 0.543 \\
Length-of-stay in the PICU (days), median (IQ) & $7(4-10)$ & $9.5(5-19)$ & $\mathbf{0 . 0 4 9}$ \\
Days of MV, median (IQ) & $5(2-8)$ & $7(3-15.5)$ & 0.077 \\
Female sex, number (\%) & $57(40 \%)$ & $10(50 \%)$ & 0.402 \\
\hline
\end{tabular}

PIM2: Pediatric Index of Mortality 2; MV: mechanical ventilation; PICU: Pediatric Intensive Care Unit; UEX: unplanned extubations. 
Patient age has been considered a risk factor for UEX., ${ }^{2,7}$ In our PICU, no statistically significant differences were found with respect to the age of patients with UEX when compared to those who did not have an episode of UEX.

There are several opinions regarding the time of the day when most UEX take place.
Some authors claim that there are no differences between day and night, while others indicate that UEX are more common during the day because patients are subjected to more procedures, or during the night because there is a lower level of surveillance. ${ }^{2,6,7,14-16}$ In this PICU, the highest number of UEX (41\%) occurred in the morning,

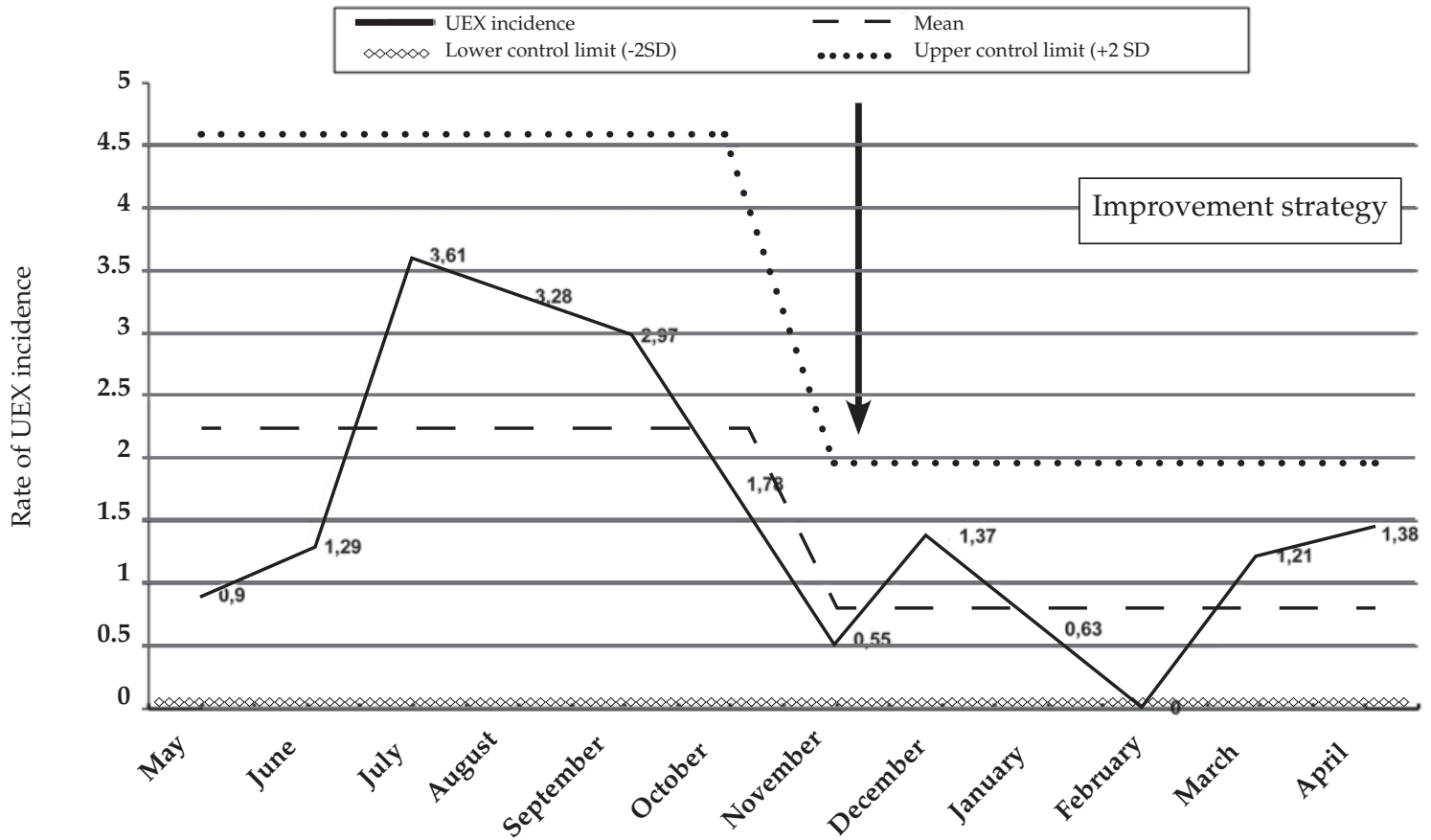

Month of the year 2010-2011

TABLE 2. Demographic characteristic of patients admitted to the PICU who required mechanical ventilation for more than 12 hours before and after the implementation of the improvement strategy

\begin{tabular}{lccc}
\hline & $\begin{array}{c}\text { Before } \\
(\boldsymbol{n = 1 6 2})\end{array}$ & $\begin{array}{c}\text { After } \\
(\boldsymbol{n}=\mathbf{1 0 0})\end{array}$ & $p$ \\
\hline Age (months), median (IQ) & $7(1-27)$ & $9(4-33.5)$ & 0.189 \\
Weight (kg), median (IQ) & $7.85(5-12)$ & $8.4(5.1-13)$ & 0.429 \\
Female sex (\%) & 41.4 & 49 & 0.226 \\
PIM2, median (IQ) & $3.6(1.3-8.4)$ & $7(2.7-16.2)$ & $\mathbf{0 . 0 0 0 7}$ \\
$\begin{array}{l}\text { Days of MV, median (IQ)) } \\
\text { Length-of-stay in PICU (days), }\end{array}$ & $5(2-8)$ & $5(2-8.5)$ & 0.983 \\
median (IQ) & $7(4-11)$ & $7.5(4-14.5)$ & 0.330 \\
Rate of UEX incidence, & & & $\mathbf{0 . 0 3 7}$ \\
mean (95\% CI) & $2.30(1.12-3.49)$ & $0.86(0.27-1.44)$ & \\
\hline
\end{tabular}

PIM2: Pediatric Index of Mortality 2; MV: mechanical ventilation; PICU: Pediatric Intensive Care Unit; IQ: interquartile range; $95 \%$ CI: $95 \%$ confidence interval. 
consistent with the time when more procedures of care are performed. Patient hygiene was part of the morning nurse shift routine (6:00 AM-11:59 AM).

The incidence of reintubation from different studies varied between $33.3 \%$ and $78 \%$. $2,3,7,11,14-19$

The bibliography has considered that UEXs during MV weaning potentially require fewer reintubations than those which occur during complete ventilation support.2,3,7,15,17,18,20,21 In this PICU, the incidence of reintubation was high, and this is because most UEX occurred during complete ventilation support, in patients with no resolution of the cause leading to MV requirement, or with an inadequate level of sedation to maintain a spontaneous ventilation.

The implementation of continuous quality improvement programs that include staff training, improved communication between healthcare professionals, standardization of procedures of care, implementation of MV weaning and sedation and analgesia protocols, and the identification of patients at a higher risk of UEX have proven to reduce its incidence. ${ }^{2,4,7,9,10}$ There are few studies assessing improvement strategies to reduce UEX incidence in the pediatric population. ${ }^{2,4,7,10}$ Da Silva, et al. ${ }^{2}$ implemented a continuous quality improvement program aimed at reducing the incidence of UEX, including: staff training, procedure standardization, identification of risk patients, and conducting a sedation and analgesia protocol. Following the program implementation, the incidence of UEX decreased from 2.9 per 100 days of MV to 0.6 per 100 days of MV ( $p=0.0001)$. Rachman, et al. ${ }^{4}$ incorporated a continuous improvement program comprised of staff training, establishing standards for ETT fixation, and ETT fixation monitoring, and they also observed a reduction in the incidence of UEX from 6.4 per 100 days of MV to 1 per 100 days of MV ( $p=0.04)$. On their side, Sadowski, et al. ${ }^{7}$ used an improvement program that consisted of: staff training, MV weaning protocol, and sedation and analgesia protocol for patients on MV. During the implementation of the program, the incidence of UEX decreased from 1.6 per 100 days of MV to 0.6 per 100 days of MV.

During the quality improvement intervention, the incidence of UEX was $63 \%$ lower, also consistent with the bibliography. $2,4,7,15$

This research has limitations, and the most significant one is that it is a single-center study. In addition, it should not be ruled out the change in the staff's behavior during the second part of the research due to their awareness of being actively observed (Hawthorne effect). Furthermore, the increased severity in patients during the second stage of the research could have led to the reduction in the incidence of UEX, either because patients are under a more strict surveillance or because they have a reduced capacity to cause a self-extubation. Therefore, there is no certainty that results are associated with the implemented intervention, given the lack of a control group. Finally, the study does not evaluate the persistence of the benefit over time.

\section{CONCLUSIONS}

The most common causes of UEX at the HGNPE's PICU were incorrect ETT fixation and performance of a procedure of care. The implementation of a quality improvement intervention consisting of training the PICU staff and standardization of ETT fixation and monitoring reduced the incidence of UEX.

\section{REFERENCES}

1 Da Silva PSL, de Carvalho WB. Unplanned extubation in pediatric critically ill patients: A systematic review and best practice recommendations. Pediat Crit Care Med 2010;11(2):287-94.

2. Da Silva PSL, de Aguilar VE, Neto HM, de Carvalho WB. Unplanned extubation in a pediatric intensive care unit: Impact of a quality improvement programme. Anaesthesia 2008;63(11):1209-16.

3. Piva JP, Amantéa S, Luchese S, Giugno K, et al. Extubacáo acidental em uma unidade de terapia intensiva. J Pediatr (Río J) 1995;71(2):72-6.

4. Rachman BR, Watson R, Woods N, Mink RB. Reducing unplanned extubations in pediatric intensive care unit: A systematic approach. Int J Pediatr 2009;2009:820495.

5. Little LA, Koening JC Jr, Newth CJ. Factors affecting accidental extubations in neonatal and pediatric intensive care. Crit Care Med 1990;18(2):163-5.

6. Curry K, Cobb S, Kutash M, Diggs C. Caracteristics associated with unplanned extubations in a surgical intensive care unit. Am J Crit Care 2008;17(1):45-51.

7. Sadowski R, DechertRE, Bandy KP, JunoJ, et al. Continuous quality improvement: Reducing unplanned extubations in pediatric intensive care unit. Pediatrics 2004;114(3):628-32.

8. Richmond AL, Jarog DL, Hanson VM. Unplanned extubation in adult critical care: Quality improvement and education payoff. Crit Care Nurse 2004;24(1): 32-7.

9. Chiang AA, Lee KC, Lee JC, Wei CH. Effectiveness of a continuous quality improvement program aiming to reduce unplanned extubation: A prospective study. Intensive Care Med 1996;22(11):1269-71.

10. Popernack ML, Thomas NJ, Lucking SE. Decreasing unplanned extubations: Utilization of the Penn State Children's Hospital sedation algorithm. Pediatr Crit Care Med 2004;5(1):58-62.

11. Hartaman ME, Macrory DC, Schulman SR. Efficacy of sedation regimens to facilitate mechanical ventilation in the pediatric intensive care unit. A systematic review. Pediatr Crit Care Med 2009;10(2):246-55. 
12. Newth CJL, Venkataraman S, Wilson DF, Meert KL, et al. Weaning and extubation readiness in pediatric patients. Pediatr Crit Care Med 2009;10(1):1-11.

13. Sessler CN. Unplanned extubations: Making progress using CQI. Intensive Care Med 1997;23(2):143-5.

14. Ream RS, Mackey K, Leet T, Green MC, et al. Association of nursing workload and unplanned extubations in a pediatric intensive care unit. Pediatr Crit Care Med 2007;8(4):366-71.

15. Bouza C, Garcia E, Diaz M, Segovia E, Rodríguez I. Unplanned extubation in orally intubated medical patients in the intensive care unit: A prospective cohort study. Heart Lung 2007;36(4):270-6.

16. Coppolo DP, May JJ. Self-extubations: A 12-month experience. Chest 1990;98(1):165-9.
17. Atkins PM, Mion LC, Mendelson W, Palmer RM, et al. Characteristics and outcome of patients who self-extubate from ventilatory support: a case-control study. Chest 1997;112(5):1317-23.

18. Phoa LL, Pek WY,Syap W, Johan A. Unplanned extubation: a local experience. Singapore Med J 2002;43(10):504-8.

19. Listello D, Sessler NC. Unplanned extubation clinical predictors for reintubation. Chest 1994;105(5):1496-503.

20. Krinsley JS, Barone JE. The drive to survive: unplanned extubation in the ICU. Chest 2005;128(2):560-6.

21. Whelan J, Simpson SQ, Levy H. Unplanned extubation. Predictors of successful termination of mechanical ventilatory support. Chest 1994;105(6):1808-12. 
- Medical record:

\section{ANNEX 1. DATA COLLECTION RECORD}

- First and last name:

- Age:

- Gender:

- Reason for admission to the PICU:

- PIM2:

- No. of UEX:

- Date of UEX:

- Nurse shift during which UEX occurred:

\begin{tabular}{l|l}
\hline 6:00 AM-11:59 AM & \\
\hline 12:00 PM-5:59 PM & \\
\hline 6:00 PM-11:59 PM & \\
\hline 0:00 AM-5:59 AM & \\
\hline Weekends and holidays 0:00 AM-11:59 AM & \\
\hline Weekends and holidays 12:00 PM-11:59 PM & \\
\hline
\end{tabular}

- Nurse-patient ratio at the time of UEX:

- Level of sedation as per the Ramsay score at the time of UEX:

- Risk factors associated with UEX:

Level 1 Patient agitated, anxious or restless

Level 2 Patient cooperative, oriented and tranquil

Level 3 Patient asleep, responsive to commands

Level 4 Patient asleep, with brisk response to stimuli (light and noise)

Level 5 Patient asleep, with response only to pain

Level 6 Unresponsive

- Reintubation:

yes

no

\begin{tabular}{l|l|l} 
& \multicolumn{1}{|c|}{ yes } & no \\
\hline Weaning & & \\
\hline Complete ventilation support & & \\
\hline Transfer & & \\
\hline Incorrect fixation of ETT & & \\
\hline Abundant secretions & & \\
\hline Procedure of care & & \\
\hline
\end{tabular}

- Days of MV:

- Length of stay in PICU (days):

- Discharge from PICU: 


\section{ANNEX 2. PACKAGE OF MEASURES}

\section{Training the PICU staff}

Courses were provided with the purpose of:

- Reporting the results of the first stage of the research.

- Creating awareness regarding the relevance and consequences of UEX.

- Disseminating the content of the package of improvement measures and how to implement it.

Courses were mandatory, aimed at all PICU staff members (doctors, nurses, and kinesiologists), they lasted 30 minutes and were given during all on-call and nurse shifts of the week.

The following materials were used for the development of the course:

a. Audiovisual presentation with graphs and tables to show a summary of the research results and the development of improvement measures to be implemented.

b. Video done at the PICU depicting the new technique for endotracheal tube (ETT) fixation.

Persons in charge of the courses and monitoring attendance were Dr. Facundo Jorro Barón and Dr. Claudia Meregalli. Staff training concluded once all the PICU staff members had attended at least one of the courses. Data were collected in a record.

\section{Standardizing the endotracheal tube fixation process}

First step: The size of the endotracheal tube selected depended on the patient age.

For children aged 2 and older, the following formulas were used:

Uncuffed ETT (modified Cole formula):

ETT number $=$ age in years $/ 4+4$

Cuffed ETT (Khine formula):

ETT number $=$ age in years $/ 4+3$

There is no formula available to calculate the ETT size in children younger than 2 years old; therefore, the ETT size was selected by airway estimation according to Table 1.

TABLE 1. Calculation of endotracheal tube size in children younger than 2 years old

\begin{tabular}{cc}
\hline Age (months) & Endotracheal tube number \\
\hline$<6$ & $3.5-4$ \\
$6-24$ & $4-4.5$ \\
\hline
\end{tabular}

Second step: The depth for ETT fixation was determined.

ETT depth was calculated using the following formula:

ETT depth $(\mathrm{cm})=$ ETT number (by the corresponding formula) $\times 3$

Third step: The ETT was fixed. The person responsible for fixing the ETT was the kinesiologist on call and the nurse in charge of the patient, and they used Tensoplast Sport@.

Fixation technique:

1) Cut a strip of Tensoplast Sport $®$.

2) Dry face region and ETT with a dressing.

3) Apply one of the Tensoplast Sport ${ }^{\circledR}$ strips above the upper lip, towards the middle of the lip.

4) Pass the Tensoplast Sport ${ }^{\circledR}$ strip around the ETT twice to hold it.

5) Continue by sticking the strip on the opposite side of the upper lip to fix the tube.

Fourth step: The ETT depth was verified with a chest X-ray. The ETT should be at the level of the 2nd thoracic vertebra, at least $0.5 \mathrm{~cm}$ from the carina. 
400 / Arch Argent Pediatr 2013;111(5):391-397 / Original article

Fifth step: Once the ETT correct fixation was confirmed, a record was completed.

This record contained the following data:

- Patient identification

- ETT number

- ETT depth

This record was placed at the head of the patient's bed.

Data was also collected in the nurse practice records.

\section{Standardizing the monitoring of ETT fixation.}

The correct ETT fixation was verified once per nurse shift and before washing the patient. The person responsible for the monitoring was the nurse in charge of the patient. Data were collected in the nurse practice record.

Fixation was modified as follows:

- If fixation was loose or soil.

- If it was necessary to modify the ETT depth. 